\title{
Recurrent Spinal Meningioma: A Case Report
}

\author{
Hoi Jung Choi, Sung Hwa Paeng, Sung Tae Kim, Yong Tae Jung \\ Department of Neurosurgy, Busan Paik Hospital, Inje University College of Medicine, Busan, Korea
}

Meningiomas are the second most common intradural spinal tumors accounting for $25 \%$ of all spinal tumors. Being a slow growing and invariably benign tumor, it responds favorably to surgical excision. In addition, spinal meningioma has low recurrence rates. However, we experienced a case of intradural extramedullary spinal meningioma which recurred 16 years after the initial surgery on a 64-year-old woman. She presented with progressive neurological symptoms and had a surgical history of removal of thoracic spinal meningioma 16 years ago due to bilateral low leg weakness. She underwent a second operation at the same site and a pale yellowish tumor was excised, which was histopathologically confirmed as meningothelial meningioma, compared with previously transitional type. she showed neurological recovery after the operation. We, therefore, report the good results of this recurrent intradural spinal meningioma case developed after 16 years with literature review.

Key Words: Spinal meningioma $\cdot$ Recurrence

\section{INTRODUCTION}

Meningiomas are the second most common intradural spinal tumors, accounting for $25 \%$ of all spinal tumors ${ }^{14}$. Being a slow growing and invariably benign tumor, it responds favorably to surgical excision. In addition, spinal meningioma has low recurrence rates. Risk factors for the disease recurrence after surgical excision have been discussed in many literature ${ }^{7,8,9,14)}$. In this report, we describe a patient with intradural extramedullary spinal meningioma that recurred 16 years after the initial surgery.

\section{CASE REPORT}

A 64-year-old woman presented with weakness in both lower limbs, which had gradually progressed over the previous 4 months, and difficulty in walking on admission. She had no sensory dysfunctions or visceral involvements, but had a surgical history (the removal of thoracic spinal tumor) for a similar episode of bilateral lower leg weakness 16 years ago (Fig. 1).

- Received: April 30, 2012 - Revised: September 21, 2012

- Accepted: September 25, 2012

Corresponding Author: Sung Hwa Paeng, MD, PhD

Department of Neurosurgy, Busan Paik Hospital, 633-165 Kaikeum-Dong, Busanjin-Ku, Busan 614-735, Korea

Tel: +82-51-890-6144, Fax: +82-51-898-4244

Email: shpaeng@empas.com
Clinical examination revealed a tender linear scar extending from the C7 to T9 spinous process. Neurological assessment revealed bilaterally increased muscle tone, associated with symmetrical paraparesis (3/5). No sensory loss or bladder/ bowel involvement was evident. Magnetic resonance images revealed at $\mathrm{Tl}$ and $\mathrm{T} 2$; Godolinium enhanced image showed a well-enhanced tumor mass on the left side in the spinal canal and marked the compression of the spinal cord combined with compressive myelopathy (Fig. 2).

She underwent a second surgery at the same site and a pale yellowish tumor was excised. The pathological examination

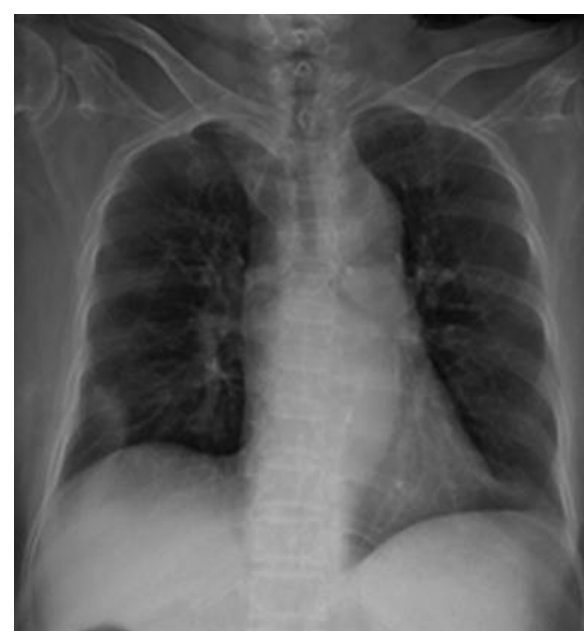

Fig. 1. Thoracic anteroposterior view showing total laminectomy state before second operation. 

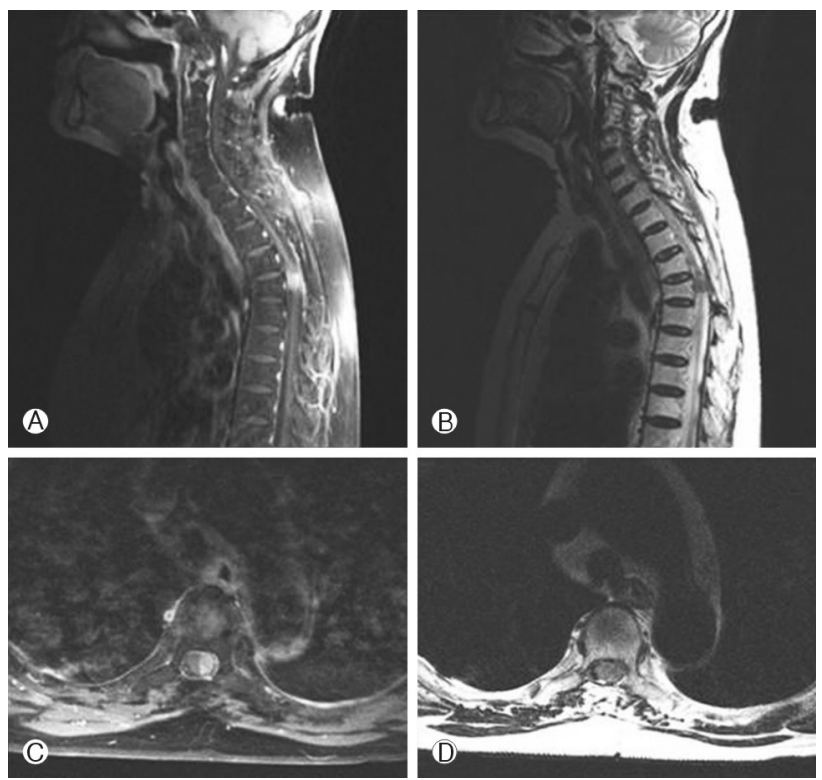

Fig. 2. Gadolinium enhanced sagittal (A) and axial (C) magnetic resonance images showing the well enhanced tumor mass of left side in spinal canal and marked compression of the spinal cord. T2 weighted sagittal (B) and axial (D) magnetic resonance images of the affected region showing the tumor is posterior and left to the spinal cord.

confirmed that it was psammomatous meningioma (Fig. 3), compared with previously transitional type (microscopic examination showed nests or concentric whorls of menigothelilal cells revealing polygonal or oval cells with eosinophilic and indistinct bordered cytoplasm and round nuclei, also showing of frequent psammoma bodies in the center of the tumor whorls). The patient showed neurological recovery after the operation. Postoperatively, the patient's sensory parameters and bladder control improved. After 2 weeks, she showed a slight improvement of motor power and was discharged.

\section{DISCUSSION}

Meningioma is a common type of tumor that accounts for $25-46 \%$ of spinal neoplasms ${ }^{8}$. The progress of spinal meningioma appeared to be more benign than its intracranial counterpart. Its prognosis is known to be favorable and the disease recurrence is also rare if the tumor is totally removed. In general, the reported rates of recurrence are ranged from $4 \%$ to $10 \%{ }^{7,3,4,14)}$. Most patients included in these recurrence results were older than 50 years. Furthermore, most results have been reported good functional outcomes in $74 \%$ to $85 \%$ of operated cases.

However, Cohen-Gadol et al. ${ }^{1)}$ reported that a recurrence rate was as high as $22 \%$ in younger patients with age less than

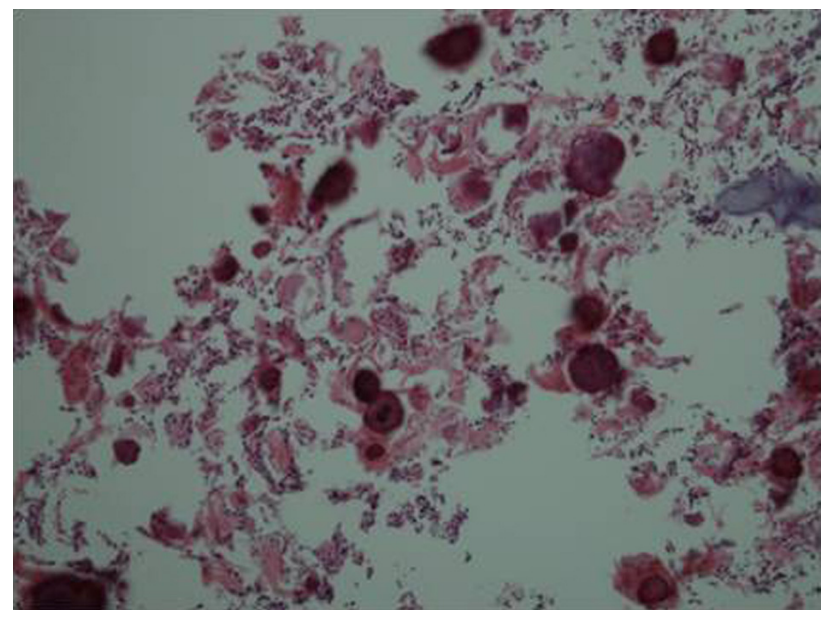

Fig. 3. The neoplasm was wholly composed of calcified psammoma bodies with a few intervening fibroblastic cells $(H \& E, \times 200)$.

50 years old. This study result showed that younger patients with the disease recurrence had aggressive histology, more extradural extension and longer follow-up periods. Therefore, younger age at the time of the first surgery could be a risk factor for recurrence in spinal meningioma.

Mirimanoff et al..$^{8)}$ reported that the recurrence free survival rates after total removal of meningioma during the follow-up periods of 5, 10 and 15 years were 93\%, 80\% and 68 $\%$, respectively. After subtotal removal of meningioma, the progression free rates were reported to be $63 \%, 45 \%$ and $9 \%$, respectively, for the same follow- up periods. The most common histological features of spinal meningioma include meningotheliomatous, fibroblastic, transitional and psammomatous. Meningotheliomatous and psa- mmomatous types are reported to be histopathologically predominant ${ }^{4,6,8)}$.

The other risk factors for the tumor recurrence are calcified meningioma and residual tumor remnants ${ }^{12)}$. In a previous study, it was reported that histologically classified psammomatous meningioma had a low risk of aggressive growth and recurrence ${ }^{11)}$. Doita et al..$^{2)}$ reported a case of psammomatous type of spinal meningioma which recurred 33 years after the surgery. In this case, a calcified recurrent spinal tumor was detected on a plain radiograph at the same site after an initial operation and preoperative paraplegia improved after the surgery.

Nadkarni et al. ${ }^{9)}$ reported a similar case as ours, which described a recurrence 18 years after the initial surgery. It was revealed that the tumor was recurrent psammomatous meningioma. Surgical removal of the tumor resulted in complete neurological recovery ${ }^{9}$. It may suggest that psammomatous histological type associated with calcification is a risk factor for recurrent spinal meningioma. Our case was a histopathologically confirmed psammomatous meningioma which was 
a pathological variant most likely to be associated with calcification. However, no calcification was seen in preoperative radiography. Moreover, the first operation revealed that the tumor was a histologically transitional type with frequent psammoma body in the center of the tumor whorls. Therefore, contrary to the precedent reports in the literature, it is considered that psammomatous meningioma of our case have been transformed from the previous transitional type, of psammomatous types, to a calcification-associated psammomatous type when it recurred over the time. This suggests that the disease recurrence rate increases if histological calcification of meningioma is postoperatively manifested and if there are residual tumor remnants. This leads us to emphasize the importance postoperative histological re-examination to establish more active additional therapeutic strategies.

Spinal meningiomas were located laterally to the spinal cord or had a component that extended laterally. A posterior location was more frequent than an anterior one ${ }^{10)}$. Unlike intracranial meningiomas, there was no correlation between recurrence and the resection of dural attachment and locations ${ }^{4,5}$. Although extradural attachment of spinal meningioma has not yet been determined as a cause of recurrence, a variety of spinal meningioma is shown to increase the rates of recurrence ${ }^{13,14)}$.

\section{CONCLUSION}

Surgery is a preferred method in the treatment of spinal meningiomas owing to excellent functional improvements and low recurrence rates following surgery. Our patient has not shown any worsening of neurological symptoms during the follow-up period. A marked neurological improvement after the second surgery in this case implies that it is possible to achieve favorable surgical outcomes in the treatment of recurrent meningiomas. We intend to highlight that the factors leading to the recurrence of meningiomas after surgery include young age, subtotal re- section of the lesion, calcification and extradural attachment.

\section{REFERENCES}

1. Cohen-Gadol AA, Zikel OM, Koch CA, Scheithauer BW, Krauss WE: Spinal meningiomas in patients younger than 50 years of age: a 21-year experience. J Neurosurg 98:258-263, 2003

2. Doita M, Harada T, Nishida K, Marui T, Kurosaka M, Yoshiya S: Recurrent calcified spinal meningioma detected by plain radiograph. Spine (Phila Pa 1976) 26:E249-252, 2001.

3. FEIRING EH, BARRON K: Late recurrence of spinal-cord meningioma. J Neurosurg 19:652-656, 1962

4. Gezen F, Kahraman S, Canakci Z, Beduk A: Review of 36 cases of spinal meningioma. Spine (Phila Pa 1976) 25:727-731, 2000

5. King AT, Sharr MM, Gullan RW, Bartlett JR: Spinal meningiomas: a 20-year review. Br J Neurosurg 12:521-526, 1998

6. Klekamp J, Samii M: Surgical results for spinal meningiomas. Surg Neurol 52:552-562, 1999

7. Levy WJ Jr, Bay J, Dohn D: Spinal cord meningioma. J Neurosurg 57:804-812, 1982

8. Mirimanoff RO, Dosoretz DE, Linggood RM, Ojemann RG, Martuza RL: Meningiomas: analysis of recurrence and progression following neurosurgical resection. J Neurosurg 62:18-24, 1985

9. Nadkarni B, Arora A, Kumar S, Bhatia A: Recurrent spinal meningioma: a case report with review of the literature. J Orthop Surg 13(3):326-329, 2005

10. Gottfried ON, Gluf W, Quinones-Hinojosa A, Kan P, Schmidt $\mathrm{MH}$ : Spinal meningiomas: surgical management and outcome. Neurosurg Focus 14(6):e2, 2003

11. Philippon J, Cornu PR, Grob R, Rivierez M: Les meningiomes recidivantes. Neurochirurgie 32:54-62, 1986

12. Roux FX, Nataf F, Pinaudeau M, Borne G, Devaux B, Meder JF: Intraspinal meningiomas: review of 54 cases with discussion of poor prognosis factors and modern therapeutic management. Surg Neurol 46:458-463, 1996

13. Scott M, Ferrara VL, Peale AR: Multiple melanotic meningiomas of the cervical cord. Case report. J Neurosurg 34:555-559, 1971

14. Solero C, Fornari M, Giombini S, Lasio G, Oliveri G, Cimino C, et al: Spinal meningiomas: review of 174 operated cases. Neurosurgery 25:153-160, 1989 